\title{
In Chemico Evaluation of Tea Tree Essential Oils as Skin Sensitizers: Impact of the Chemical Composition on Aging and Generation of Reactive Species
}

Cristina Avonto, ${ }^{\dagger}$ Amar G. Chittiboyina,${ }^{\dagger}$ Mei Wang,${ }^{\dagger}$ Yelkaira Vasquez, ${ }^{\dagger}$ Diego Rua, ${ }^{\S}$ and Ikhlas A. Khan. $*,+*$

${ }^{\dagger}$ National Center for Natural Products Research; ${ }^{\dagger}$ Division of Pharmacognosy, Department of BioMolecular Sciences; School of Pharmacy, University of Mississippi, University, Mississippi 38677, USA. ${ }^{\S}$ The Center for Food Safety and Applied Nutrition, US Food and Drug Administration, 5100 Paint Branch Parkway, College Park, Maryland 20740, USA.

$\underline{\text { ikhan@olemiss.edu }}$

SUPPORTING INFORMATION 


\section{Table of Contents}

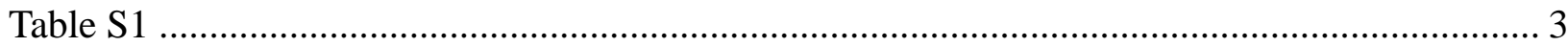

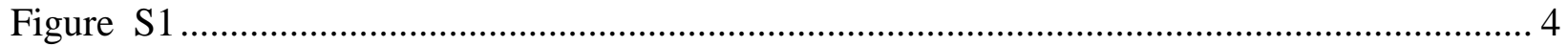

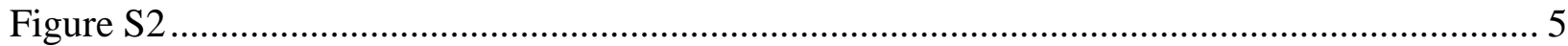

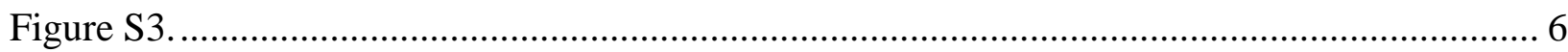

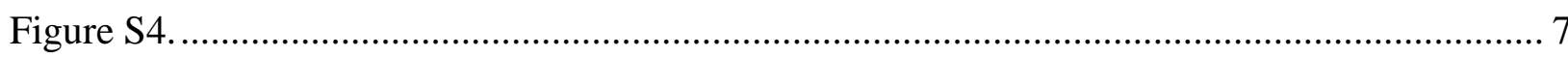

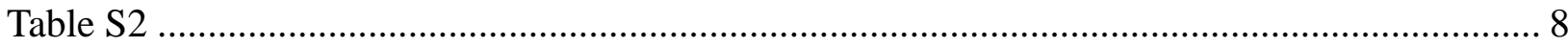

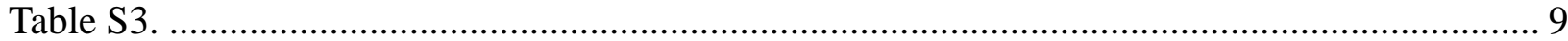

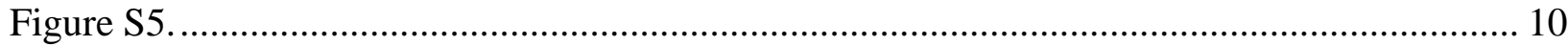

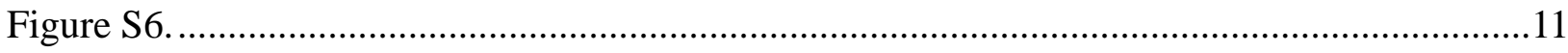

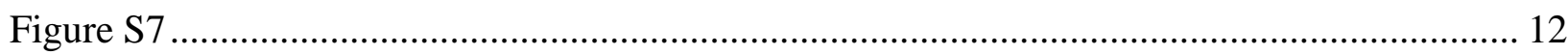

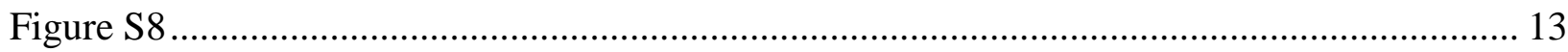

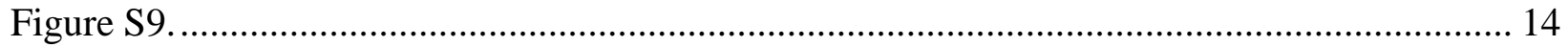

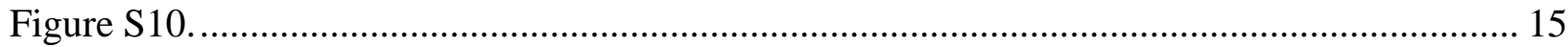

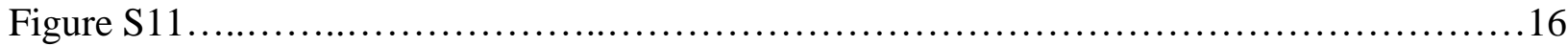


Table S1. Composition requirements of authentic TTO as defined according to ISO/FDIS 4730:2004.

\begin{tabular}{|c|c|c|c|}
\hline & COMPOUND & MIN. (\%) & MAX. (\%) \\
\hline 1 & $\alpha$-Pinene & 1 & 6 \\
\hline 2 & Sabinene & Trace & 3.5 \\
\hline 3 & $\alpha$-Terpinene & 5 & 13 \\
\hline 4 & $p$-Cymene & 0.5 & 8 \\
\hline 5 & 1,8-Cineole & Trace & 15 \\
\hline 6 & Limonene & 0.5 & 1.5 \\
\hline 7 & $\mathrm{\gamma}$-Terpinene & 10 & 28 \\
\hline 8 & Terpinolene & 1.5 & 5 \\
\hline 9 & Terpinen-4-ol & 30 & 48 \\
\hline 10 & $\alpha$-Terpineol & 1.5 & 8 \\
\hline 11 & Aromadendrene & Trace & 3 \\
\hline 12 & Viridiflorene & Trace & 3 \\
\hline 13 & $\delta$-Cadinene & Trace & 3 \\
\hline 14 & Globulol & Trace & 1 \\
\hline 15 & Viridiflorol & Trace & 1 \\
\hline
\end{tabular}

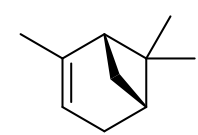

1<smiles>CC1=CC[C@](O)(C(C)C)CC1</smiles>

9<smiles>C=C1CC[C@]2(C(C)C)C[C@H]1C2</smiles>

2<smiles>CC1=CC=C(C(C)C)CC1</smiles>

3<smiles>C=C1CCC2[C@H]([C@H]3[C@@H](C)CC[C@@H]13)C2(C)C</smiles>

11<smiles>Cc1ccc(C(C)C)cc1</smiles>

4<smiles>CC1=C2CC[C@@H](C)C2[C@H]2[C@H](CC1)C2(C)C</smiles>

12

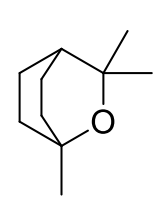

5<smiles>C=C(C)[C]1CC=C(C)CC1</smiles>

6<smiles>CC1=CCC(C(C)C)=CC1</smiles>

7<smiles>CC1=CC2C(=C(C)CC[C@@H]2C(C)C)CC1</smiles>

13<smiles>C[C@H]1CC[C@H]2[C@@H]1[C@@H]1[C@@H](CC[C@@]2(C)O)C1(C)C</smiles>

14<smiles>CC1=CCC(=C(C)C)CC1</smiles>

8<smiles>C[C@H]1CCC2[C@@H]1C1C(CC[C@@]2(C)O)C1(C)C</smiles>

15 
Figure S1. A) GC-MS profile of a typical authentic tree tea oil (T1). B. Chromatogram expanded between 16-28 min to show the baseline separation of 1-8 compounds.
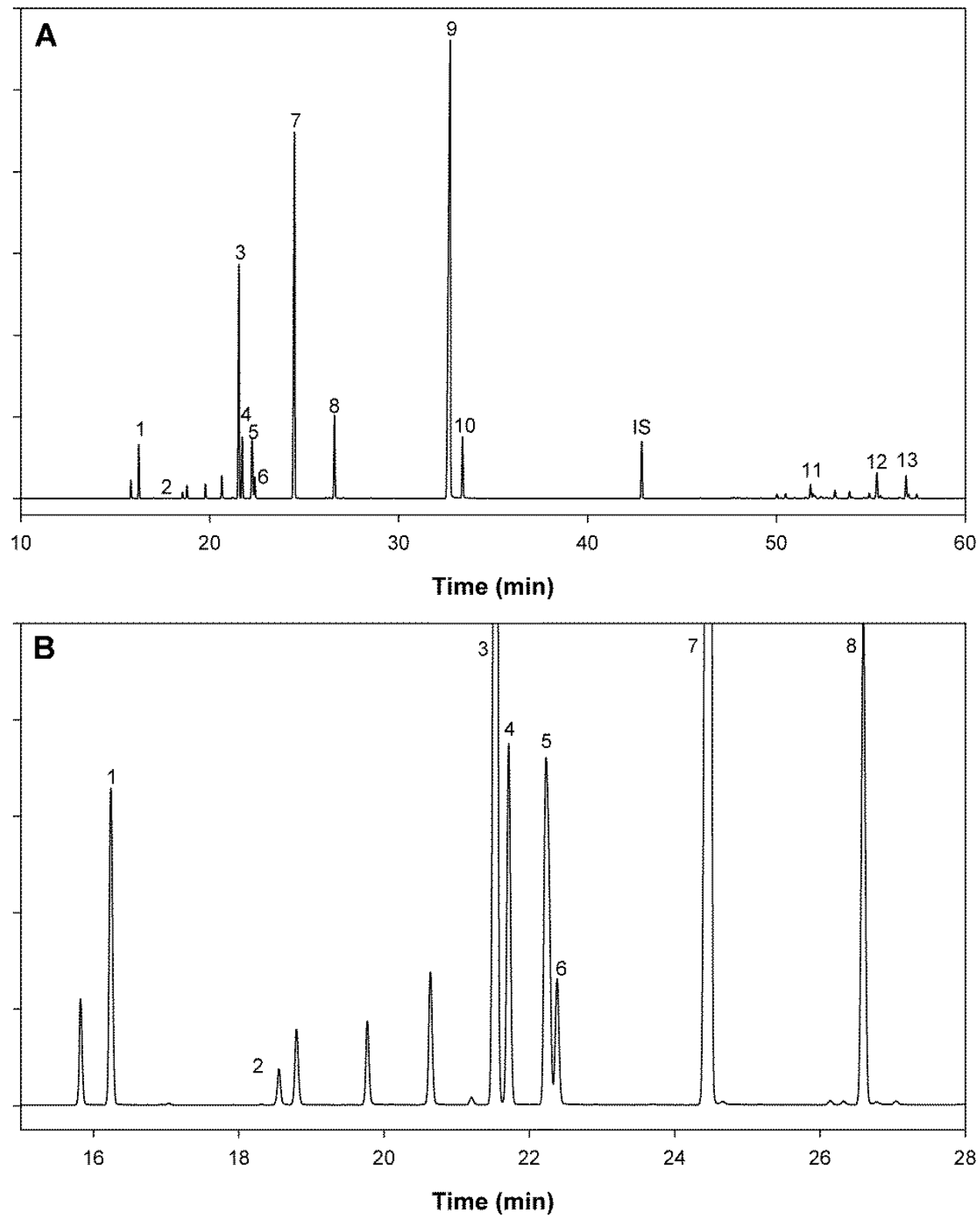
Figure S2. GC profile of TTO samples T2-T4.

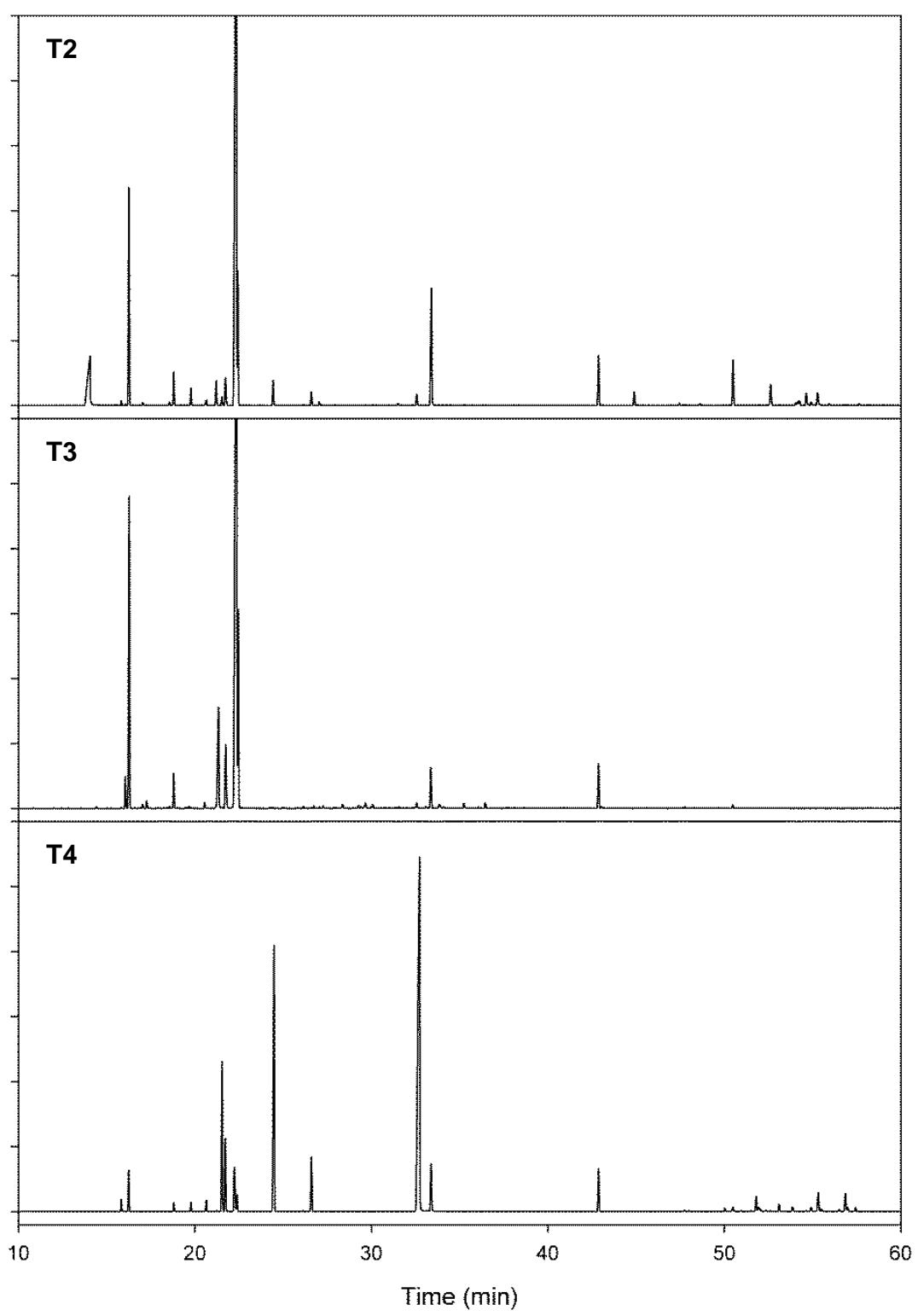


Figure S3. GC profile of TTO samples T5-T7.

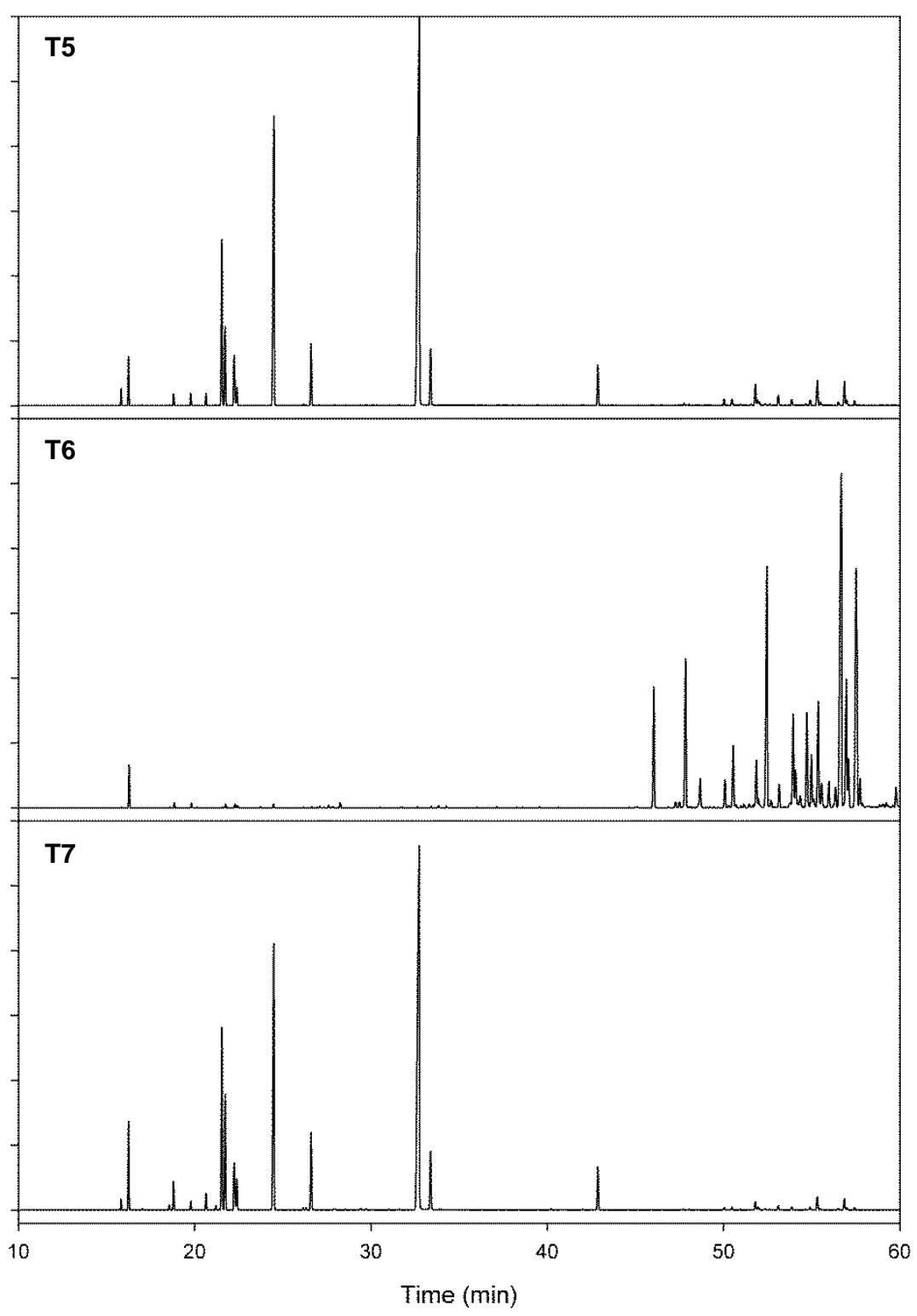


Figure S4. GC profile of TTO samples T8 -T10.

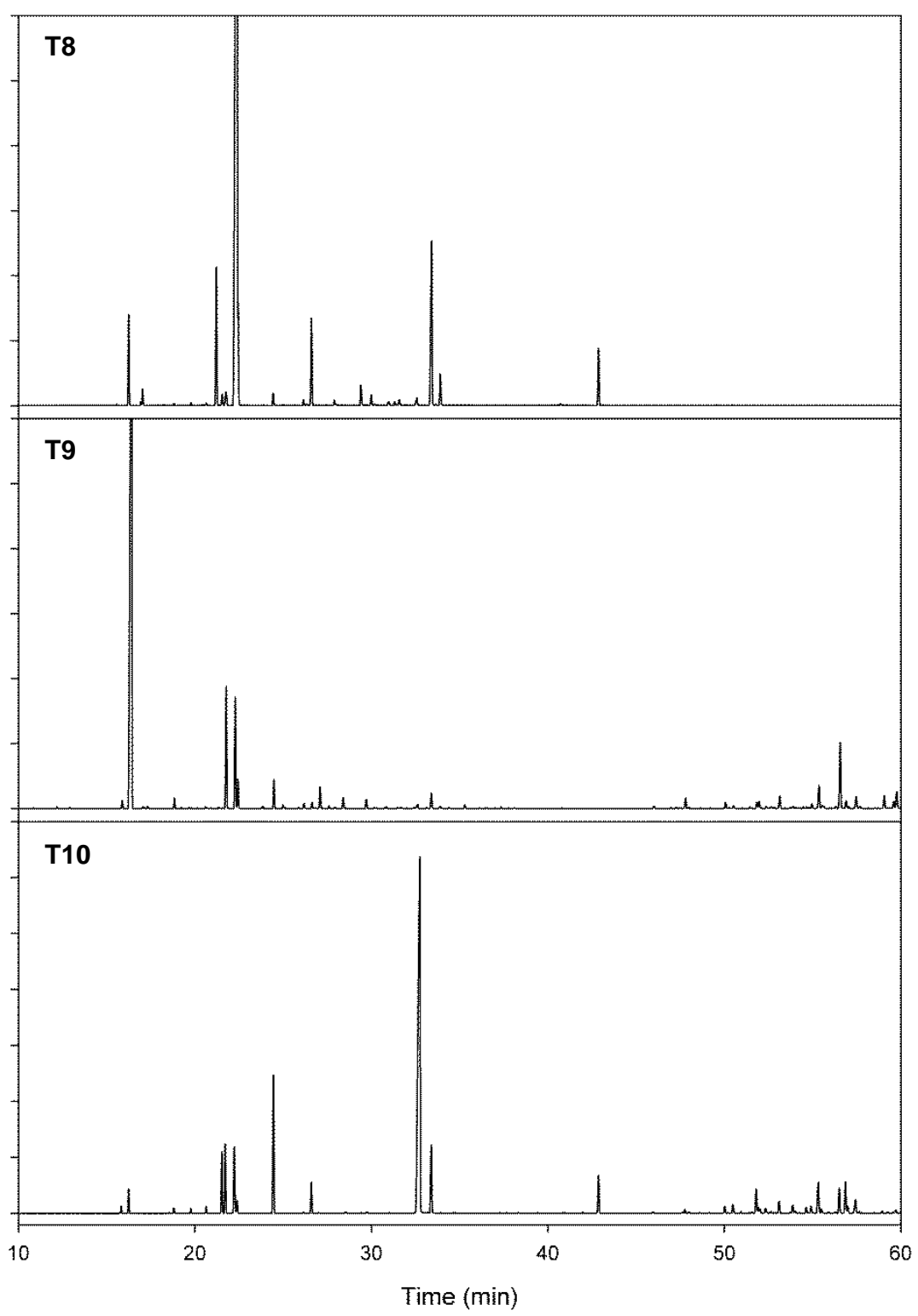


Table S2. Composition of TTO oils before aging.

\begin{tabular}{|c|c|c|c|c|c|c|c|c|c|c|c|c|}
\hline \multirow[b]{2}{*}{ \# } & \multirow[b]{2}{*}{ Compound } & \multirow[b]{2}{*}{ RT } & $\begin{array}{c}\mathrm{T} 1-M . \\
\text { alternifolia }\end{array}$ & $\begin{array}{l}\mathrm{T} 2-M \\
\text { cajeputi }\end{array}$ & $\begin{array}{l}\mathrm{T} 3-M . \\
\text { cajeputi }\end{array}$ & $\begin{array}{c}\mathrm{T} 4-M . \\
\text { alternifolia }\end{array}$ & $\begin{array}{c}\text { T5 - M. } \\
\text { linariifolia }\end{array}$ & $\begin{array}{c}\mathrm{T} 6-L . \\
\text { scoparium }\end{array}$ & $\begin{array}{c}\mathrm{T} 7-M . \\
\text { alternifolia }\end{array}$ & $\begin{array}{c}\mathrm{T} 8-M . \\
\text { viridiflora }\end{array}$ & $\begin{array}{l}\mathrm{T} 9-K . \\
\text { ericoides }\end{array}$ & $\begin{array}{l}\mathrm{T} 10- \\
\text { Leptospermum- } \\
\text { melaleuca }\end{array}$ \\
\hline & & & $\begin{array}{c}\text { Peak Area } \\
\%\end{array}$ & $\begin{array}{c}\text { Peak Area } \\
\%\end{array}$ & $\begin{array}{c}\text { Peak Area } \\
\%\end{array}$ & $\begin{array}{c}\text { Peak Area } \\
\%\end{array}$ & $\begin{array}{c}\text { Peak Area } \\
\%\end{array}$ & $\begin{array}{c}\text { Peak Area } \\
\%\end{array}$ & $\begin{array}{c}\text { Peak Area } \\
\%\end{array}$ & $\begin{array}{c}\text { Peak Area } \\
\%\end{array}$ & $\begin{array}{c}\text { Peak Area } \\
\%\end{array}$ & $\begin{array}{c}\text { Peak Area } \\
\%\end{array}$ \\
\hline 1 & $\alpha$-pinene & 16.28 & 2.08 & 9.81 & 16.19 & 2.02 & 2.06 & 0.81 & 3.73 & 3.19 & 52.78 & 1.02 \\
\hline 2 & Sabinene & 18.59 & 0.07 & 0.18 & 0.1 & 0.07 & 0.07 & 0.01 & 0.4 & - & - & 0.03 \\
\hline 3 & $\alpha$-terpinene & 21.60 & 9.14 & 0.46 & 0.02 & 8.18 & 8.85 & 0.01 & 10.44 & 0.55 & 0.05 & 3.12 \\
\hline 4 & $p$-cymene & 21.78 & 5.03 & 1.87 & 3.84 & 3.44 & 3.82 & 0.07 & 4.87 & 0.87 & 6.54 & 3.11 \\
\hline 5 & 1,8 -cineole & 22.28 & 2.84 & 47.93 & 47.75 & 2.75 & 2.81 & 0.07 & 2.68 & 67.24 & 5.99 & 3.07 \\
\hline 6 & Limonene & 22.43 & 0.81 & 5.36 & 7.74 & 0.77 & 0.79 & 0.05 & 1.33 & 0.02 & 1.5 & 0.54 \\
\hline 7 & $y$-terpinene & 24.56 & 17.03 & 1.26 & 0.06 & 16.59 & 17.64 & 0.08 & 16.57 & 0.54 & 1.5 & 7.43 \\
\hline 8 & terpinolene & 26.65 & 3.32 & 0.69 & - & 3.17 & 3.28 & 0.03 & 4.27 & 4.34 & 0.34 & 1.86 \\
\hline 9 & Terpinen-4-ol & 32.87 & 37.51 & 0.67 & - & 41.69 & 37.29 & 0.02 & 36.41 & 0.51 & 0.25 & 35.44 \\
\hline 10 & $\alpha$-Terpineol & 33.47 & 2.94 & 7.19 & 2.57 & 3.44 & 3.11 & 0.03 & 3.56 & 9.93 & 0.9 & 3.96 \\
\hline 11 & Aromadendrene & 51.85 & 1.38 & - & 0.02 & 1.84 & 1.66 & 1.34 & 0.87 & - & 0.39 & 2.34 \\
\hline 12 & Viridiflorene & 55.37 & 1.82 & 1.30 & - & 1.70 & 2.2 & 3.21 & 1.67 & - & 1.58 & 2.95 \\
\hline 13 & $\delta$-Cadinene & 56.91 & 1.88 & 0.12 & - & 1.68 & 2.07 & 3.78 & 1.17 & - & 0.49 & 2.84 \\
\hline 14 & Globulol & 60.40 & 0.41 & 0.01 & - & 0.48 & 0.49 & 0.59 & 0.32 & - & 0.17 & 1.25 \\
\hline 15 & Viridiflorol & 60.84 & 0.2 & 0.3 & - & 0.24 & 0.24 & 0.19 & 0.16 & - & 4.16 & 0.51 \\
\hline
\end{tabular}


Table S3. Variation of marker compounds over 18 months aging.

\begin{tabular}{|c|c|c|c|c|c|c|c|}
\hline \multicolumn{4}{|c|}{ T1 - M. alternifolia } & \multicolumn{4}{|c|}{ T2-M. cajeputi } \\
\hline \multicolumn{4}{|c|}{ Area $\%$} & \multicolumn{4}{|c|}{ Area $\%$} \\
\hline & Fresh & Aged & Diff. (\%) & & Fresh & Aged & Diff. (\%) \\
\hline$\alpha$-Pinene & 2.08 & 0.9 & -1.18 & $\alpha$-Pinene & 9.81 & 4.82 & -4.99 \\
\hline$\alpha$-Terpinene & 9.14 & 14.06 & 4.92 & $\alpha$-Terpinene & 0.46 & 0.59 & 0.13 \\
\hline$p$-Cymene & 5.03 & 7.23 & 2.2 & p-Cymene & 1.87 & 3.98 & 2.11 \\
\hline 1,8-Cineole & 2.84 & 2.01 & -0.83 & 1,8-Cineole & 47.93 & 51.3 & 3.37 \\
\hline $\mathrm{y}$-Terpinene & 17.03 & 17.13 & 0.1 & y-Terpinene & 1.26 & 1.03 & -0.23 \\
\hline Terpinolene & 3.32 & 4.92 & 1.6 & Terpinolene & 0.69 & 1 & 0.31 \\
\hline Terpinen-4-ol & 37.51 & 24.43 & -13.08 & Terpinen-4-ol & 0.67 & 0.39 & -0.28 \\
\hline$\alpha$-Terpineol & 2.94 & 2.02 & -0.92 & $\alpha$-Terpineol & 7.19 & 4.57 & -2.62 \\
\hline \multicolumn{4}{|c|}{ T3-M. cajeputi } & \multicolumn{4}{|c|}{ T4 - M. alternifolia } \\
\hline \multicolumn{4}{|c|}{ Area $\%$} & \multicolumn{4}{|c|}{ Area $\%$} \\
\hline & Fresh & Aged & Diff. (\%) & & Fresh & Aged & Diff. (\%) \\
\hline$\alpha$-Pinene & 16.19 & 7.64 & -8.55 & $\alpha$-Pinene & 2.02 & 0.97 & -1.05 \\
\hline$\alpha$-Terpinene & 0.02 & & -0.02 & $\alpha$-Terpinene & 8.18 & 10.31 & 2.13 \\
\hline$p$-Cymene & 3.84 & 9.08 & 5.24 & $p$-Cymene & 3.44 & 12.33 & 8.89 \\
\hline 1,8-Cineole & 47.75 & 62.7 & 14.95 & 1,8-Cineole & 2.75 & 2.19 & -0.56 \\
\hline $\mathrm{y}$-Terpinene & 0.06 & & -0.06 & y-Terpinene & 16.59 & 14.56 & -2.03 \\
\hline Terpinolene & - & - & - & Terpinolene & 3.17 & 3.98 & 0.81 \\
\hline Terpinen-4-ol & - & - & - & Terpinen-4-ol & 41.69 & 22.34 & -19.35 \\
\hline$\alpha$-Terpineol & 2.57 & - & -2.57 & $\alpha$-Terpineol & 3.44 & 1.97 & 0.81 \\
\hline \multicolumn{4}{|c|}{ T5 - M. linariifolia } & \multicolumn{4}{|c|}{ T6 - L. scoparium } \\
\hline \multicolumn{4}{|c|}{ Area $\%$} & \multicolumn{4}{|c|}{ Area \% } \\
\hline & Fresh & Aged & Diff. (\%) & & Fresh & Aged & Diff. (\%) \\
\hline$\alpha$-Pinene & 2.06 & 0.87 & -1.19 & $\alpha$-Pinene & 0.81 & 0.24 & -0.57 \\
\hline$\alpha$-Terpinene & 8.85 & 10 & 1.15 & $\alpha$-Terpinene & 0.01 & & -0.01 \\
\hline p-Cymene & 3.82 & 11.26 & 7.44 & $p$-Cymene & 0.07 & 0.11 & 0.04 \\
\hline 1,8-Cineole & 2.81 & 2.08 & -0.73 & 1,8-Cineole & 0.07 & 0.04 & -0.03 \\
\hline $\mathrm{y}$-Terpinene & 17.64 & 14.13 & -3.51 & y-Terpinene & 0.08 & 0.04 & -0.04 \\
\hline Terpinolene & 3.28 & 3.88 & 0.6 & Terpinolene & 0.03 & 0.03 & 0 \\
\hline Terpinen-4-ol & 37.29 & 23.36 & -13.93 & Terpinen-4-ol & 0.02 & - & -0.02 \\
\hline$\alpha$-Terpineol & 3.11 & 1.98 & -1.13 & $\alpha$-Terpineol & 0.03 & - & -0.03 \\
\hline \multicolumn{4}{|c|}{ T7 - M. alternifolia } & \multicolumn{4}{|c|}{ T8 - M. viridiflora } \\
\hline \multicolumn{4}{|c|}{ Area \% } & \multicolumn{4}{|c|}{ Area $\%$} \\
\hline & Fresh & Aged & Diff. (\%) & & Fresh & Aged & Diff. (\%) \\
\hline$\alpha$-Pinene & 3.73 & 1.51 & -2.22 & $\alpha$-Pinene & 3.19 & 1.59 & -1.6 \\
\hline$\alpha$-Terpinene & 10.44 & 5.13 & -5.31 & $\alpha$-Terpinene & 0.55 & 0.6 & 0.05 \\
\hline p-Cymene & 4.87 & 24.35 & 19.48 & $p$-Cymene & 0.87 & 2.23 & 1.36 \\
\hline 1,8-Cineole & 2.68 & 1.88 & -0.8 & 1,8-Cineole & 67.24 & 66.97 & -0.27 \\
\hline $\mathrm{y}$-Terpinene & 16.57 & 8.07 & -8.5 & y-Terpinene & 0.54 & 0.42 & -0.12 \\
\hline Terpinolene & 4.27 & 3.64 & -0.63 & Terpinolene & 4.34 & 5.64 & 1.3 \\
\hline Terpinen-4-ol & 36.41 & 26.46 & -9.95 & Terpinen-4-ol & 0.51 & 0.26 & -0.25 \\
\hline$\alpha$-Terpineol & 3.56 & 2.71 & -0.85 & $\alpha$-Terpineol & 9.93 & 6.8 & -3.13 \\
\hline \multicolumn{4}{|c|}{ T9 - K. ericoides } & \multicolumn{4}{|c|}{ T10 - Lema ${ }^{\circledR}$ oil } \\
\hline & & & & & & & \\
\hline & Fresh & Aged & Diff. (\%) & & Fresh & Aged & Diff. (\%) \\
\hline$\alpha$-Pinene & 52.78 & 37.59 & -15.2 & $\alpha$-Pinene & 1.02 & 0.48 & -0.54 \\
\hline$\alpha$-Terpinene & 0.05 & & -0.05 & $\alpha$-Terpinene & 3.12 & 3.49 & 0.37 \\
\hline p-Cymene & 6.54 & 14.26 & 7.72 & $p$-Cymene & 3.11 & 8.22 & 5.11 \\
\hline 1,8-Cineole & 5.99 & 5.67 & -0.32 & 1,8-Cineole & 3.07 & 2.67 & -0.4 \\
\hline $\mathrm{y}$-Terpinene & 1.5 & 0.88 & -0.62 & $\mathrm{y}$-Terpinene & 7.43 & 5.39 & -2.04 \\
\hline Terpinolene & 0.34 & - & -0.34 & Terpinolene & 1.86 & 1.94 & 0.08 \\
\hline Terpinen-4-ol & 0.25 & 0.16 & -0.09 & Terpinen-4-ol & 35.44 & 21.53 & -13.91 \\
\hline$\alpha$-Terpineol & 0.9 & 0.48 & -0.42 & $\alpha$-Terpineol & 3.96 & 2.51 & -1.45 \\
\hline
\end{tabular}


Figure S5. Quantification of main TTO constituents before aging.

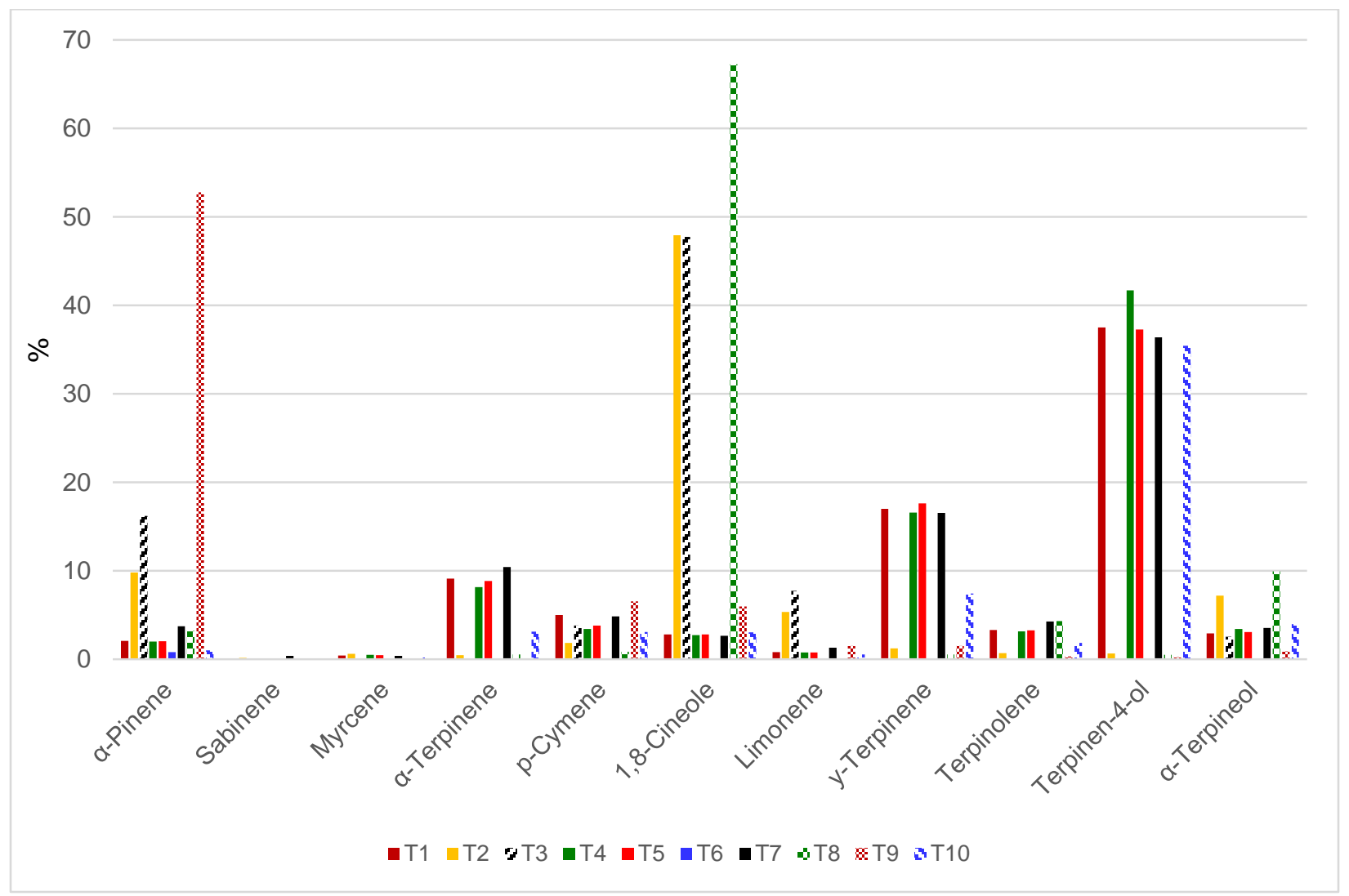


Figure S6. Quantification of peroxide content in TTO samples after 18 months storage. All samples were diluted in 3 volumes of deionized water (sample 13105 was diluted 200x, 13145 was diluted 20x).

T1

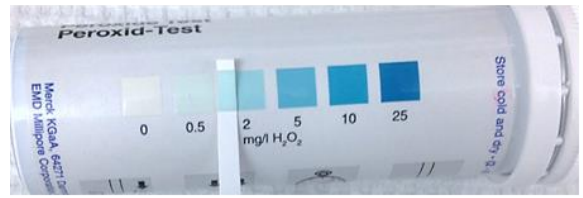

T4 (20X dil)

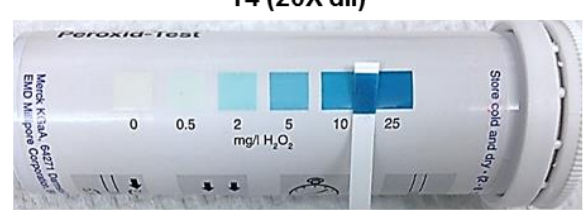

T8

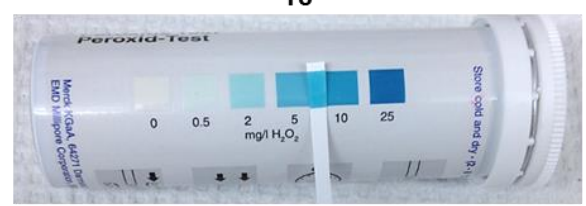

T2

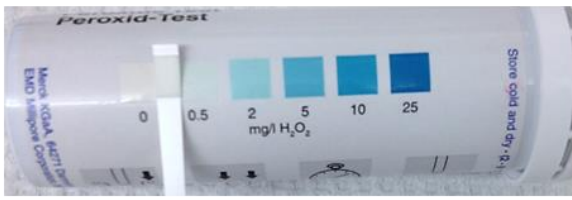

T5

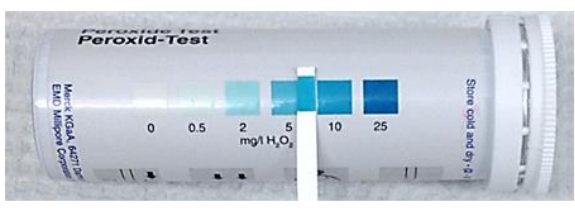

T9

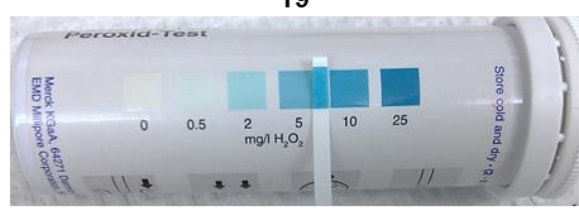

T7 (200X dil)

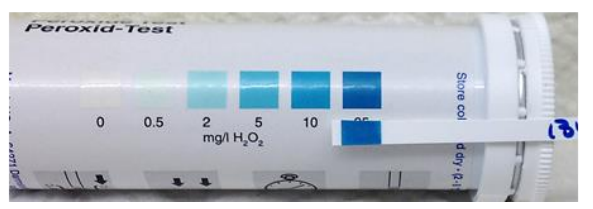

T3

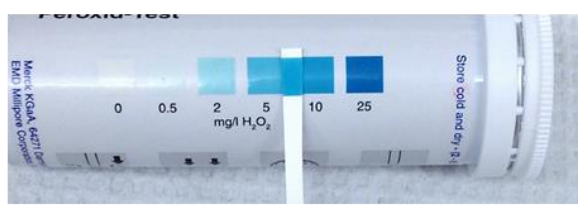

T6

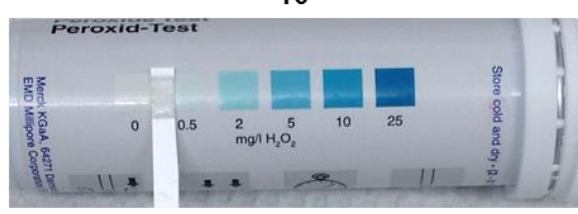

T10

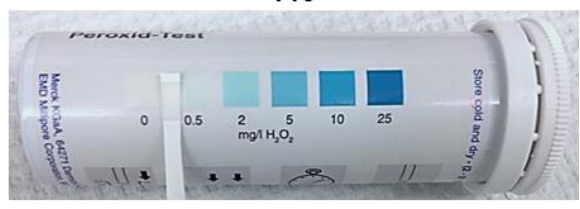


Figure S7. GC profile of pure components from TTO before and after aging (IS = Internal Standard).

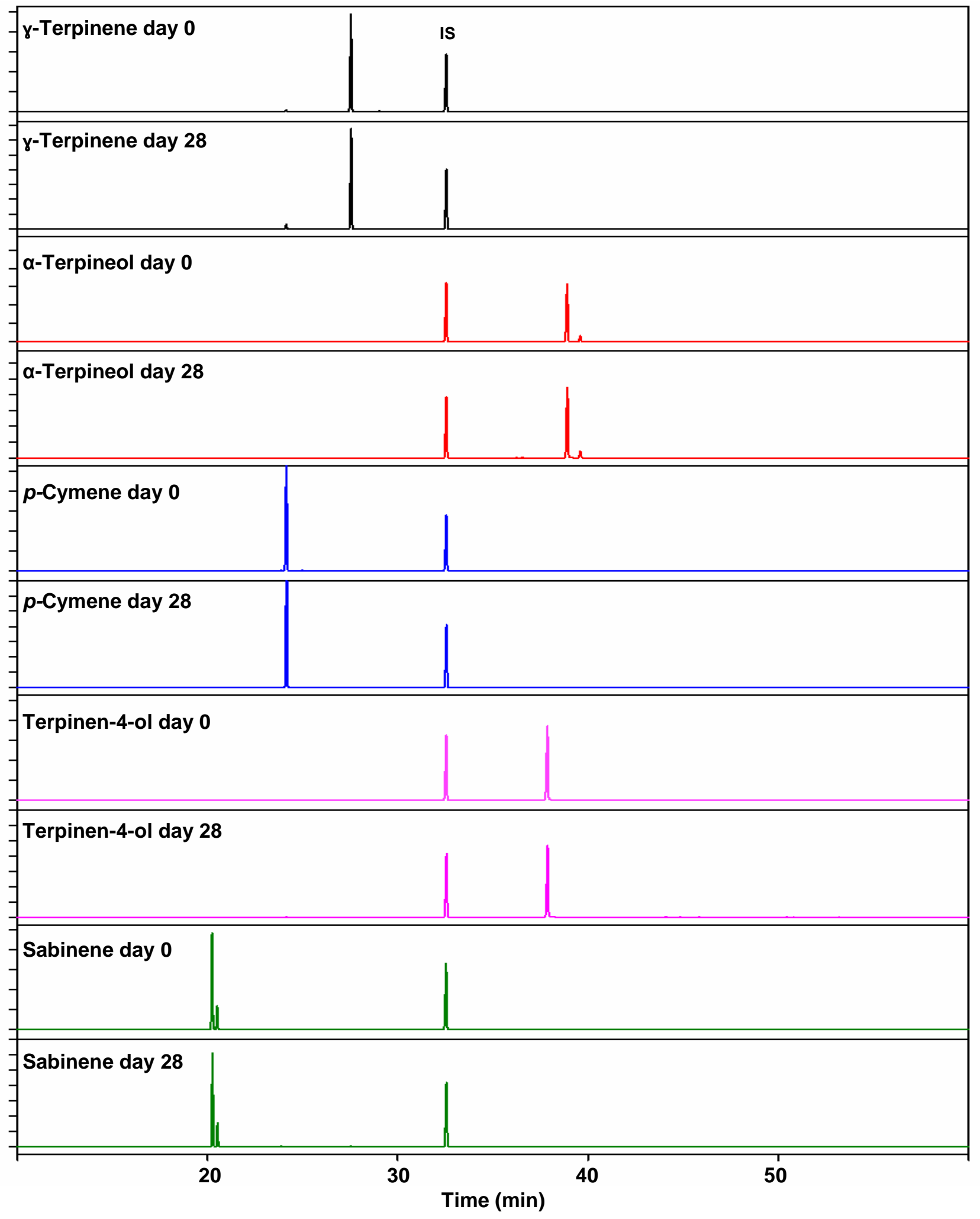


Figure S8. GC chromatogram of A) $\alpha$-terpinene and B) terpinolene upon aging (IS = Internal Standard).
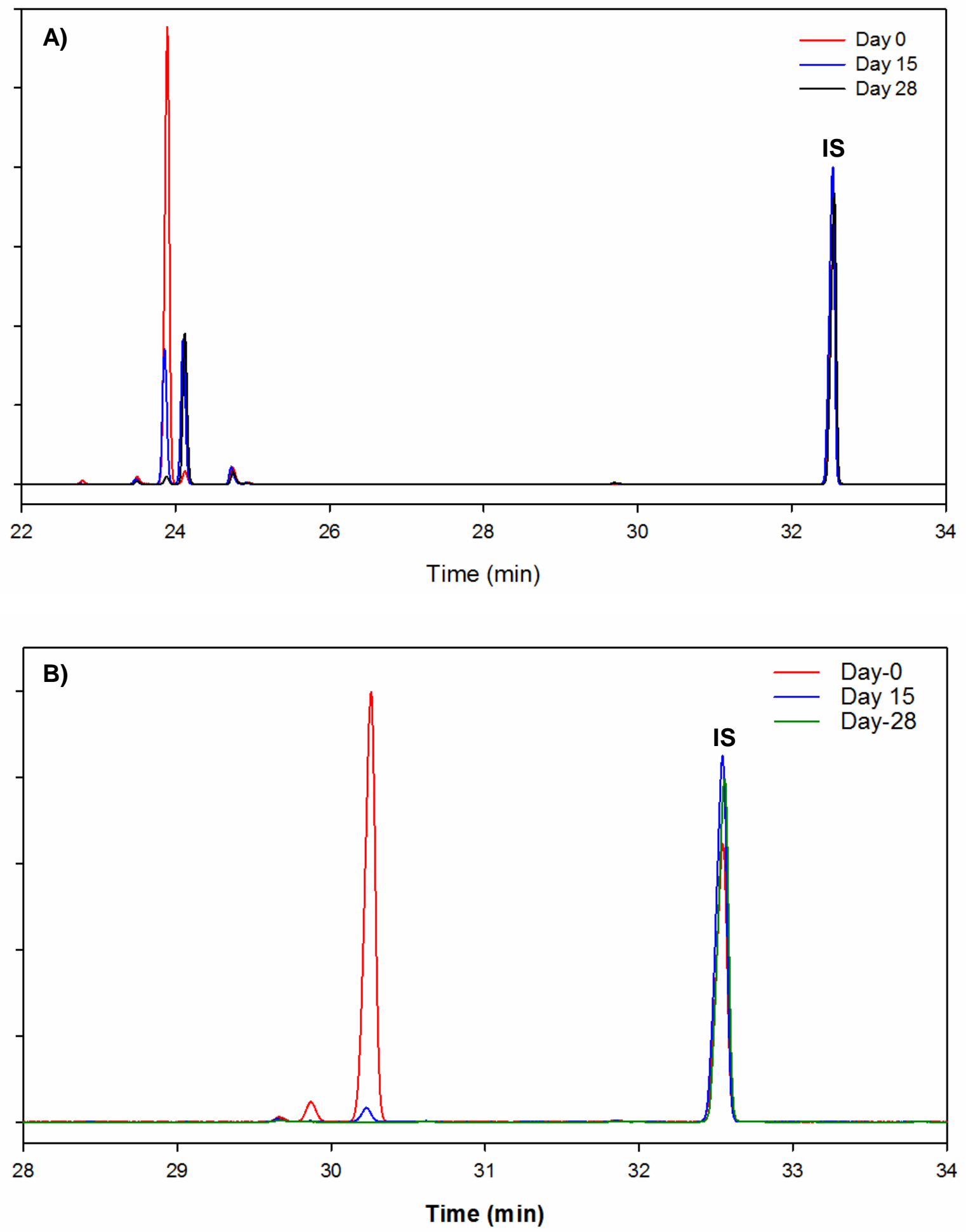
Figure S9. Quantification of peroxide content of the principal TTO constituents after accelerate aging conditions.
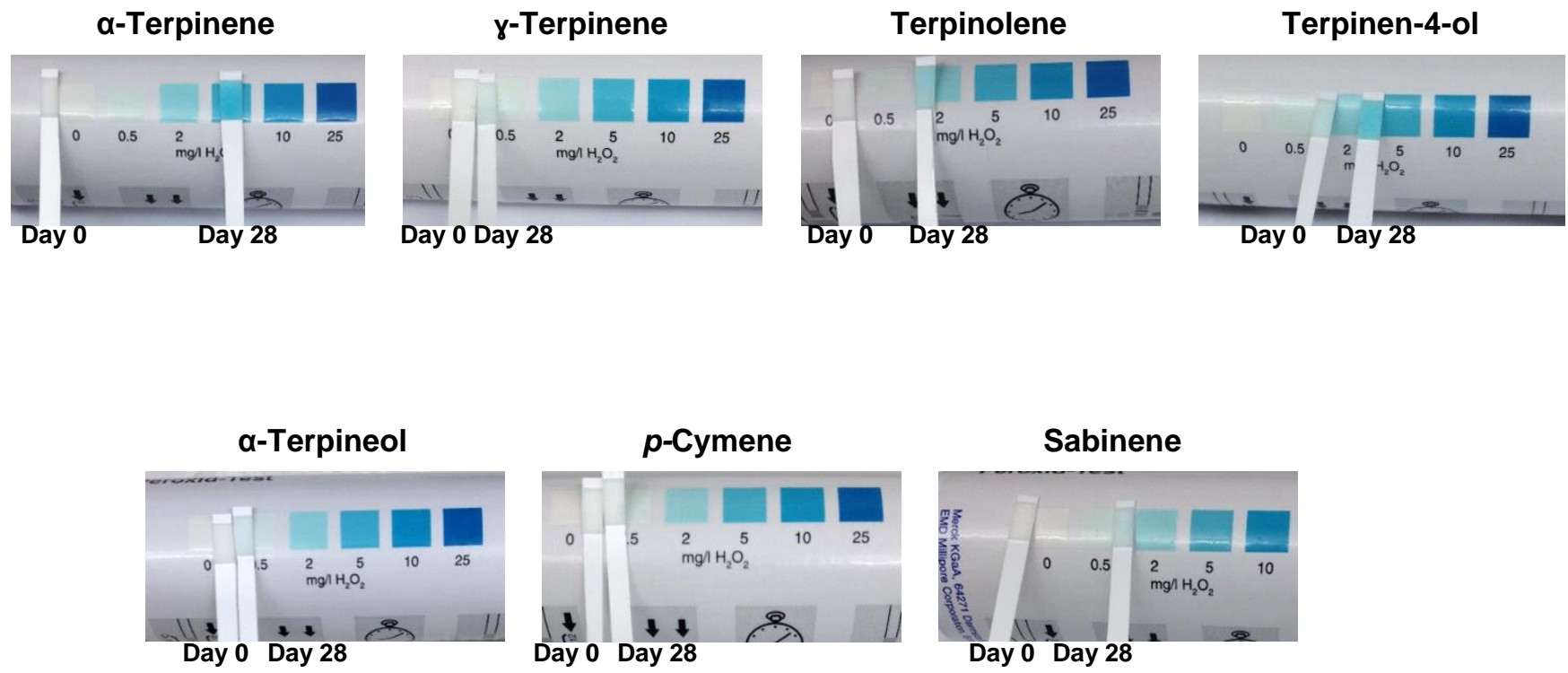
Figure S10. A) Identification of DCYA adducts A/B by UHPLC-DAD-MS. DAD profile $(340 \mathrm{~nm})$ of isolated adduct A (below) and co-injection of A with $\mathrm{T} 7$ enriched fraction after reaction with DCYA (above). B) ESI+ m/z profile of isolated adduct A.
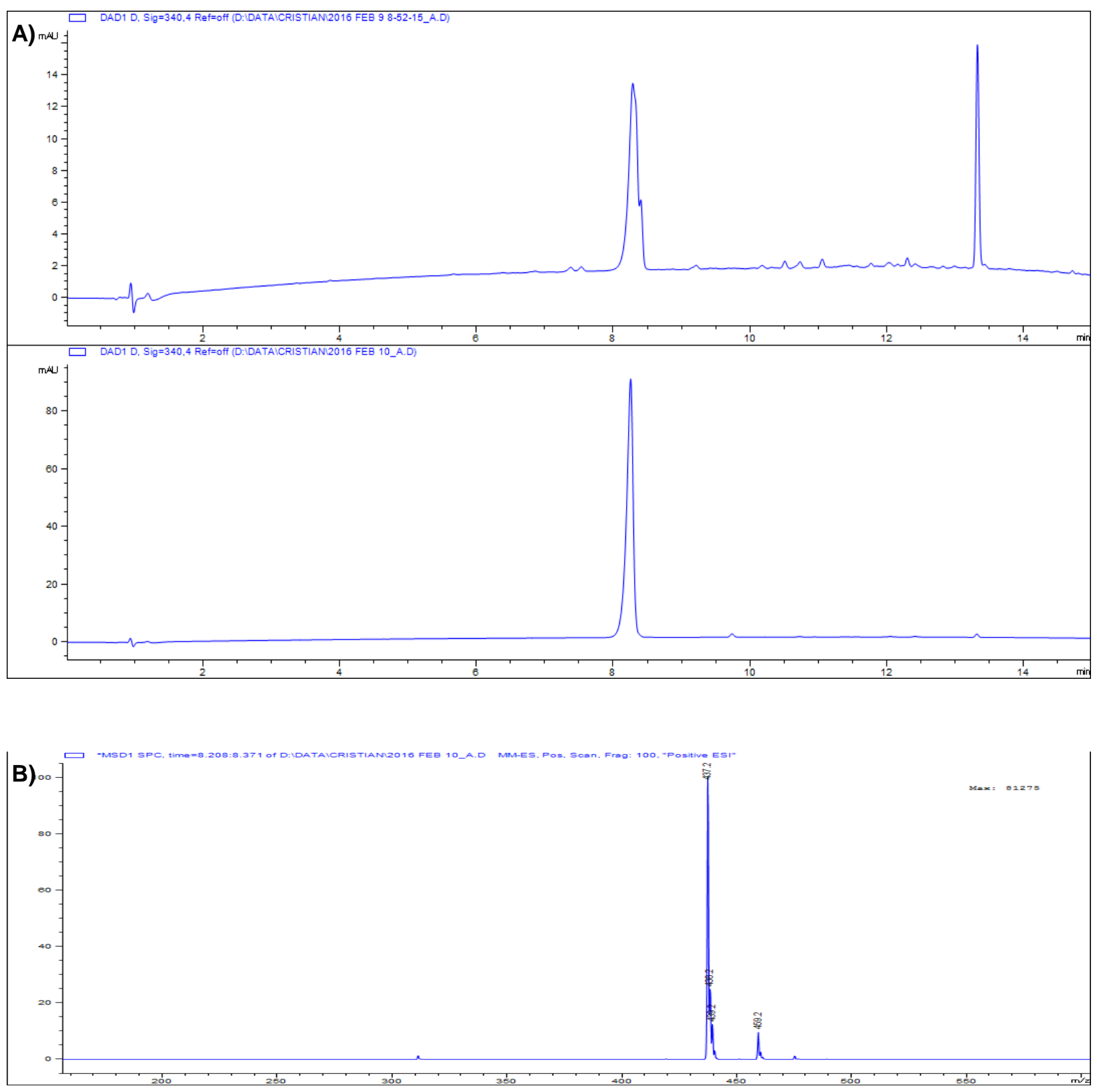
Figure S11. NMR spectra of adduct A in MeOD (5oo MHz): A) ${ }^{1} \mathrm{H}$ NMR; B) ${ }^{13} \mathrm{C}$; C) HSQCAD; D) gCOSY; E) gHMBCAD.

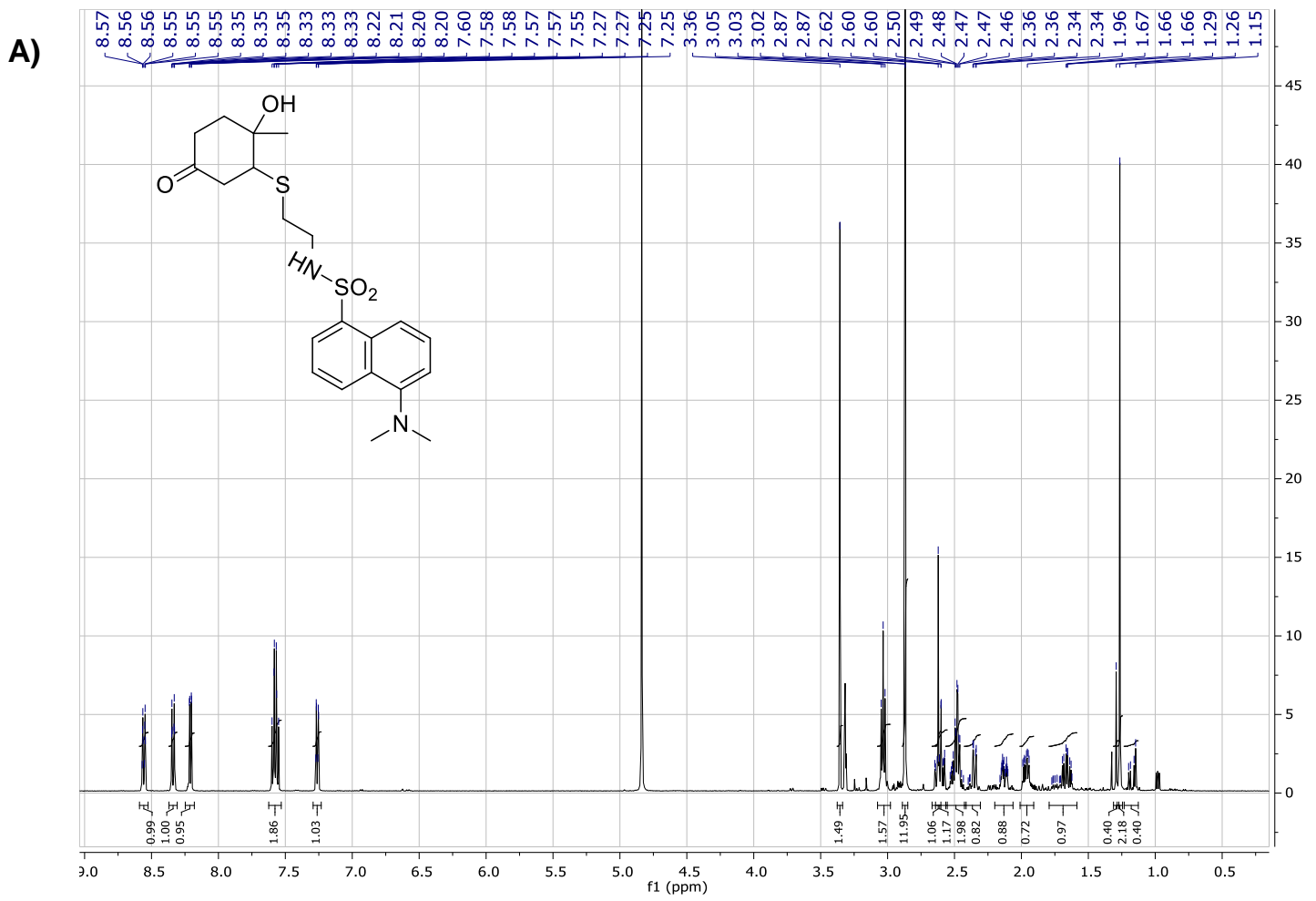

B)

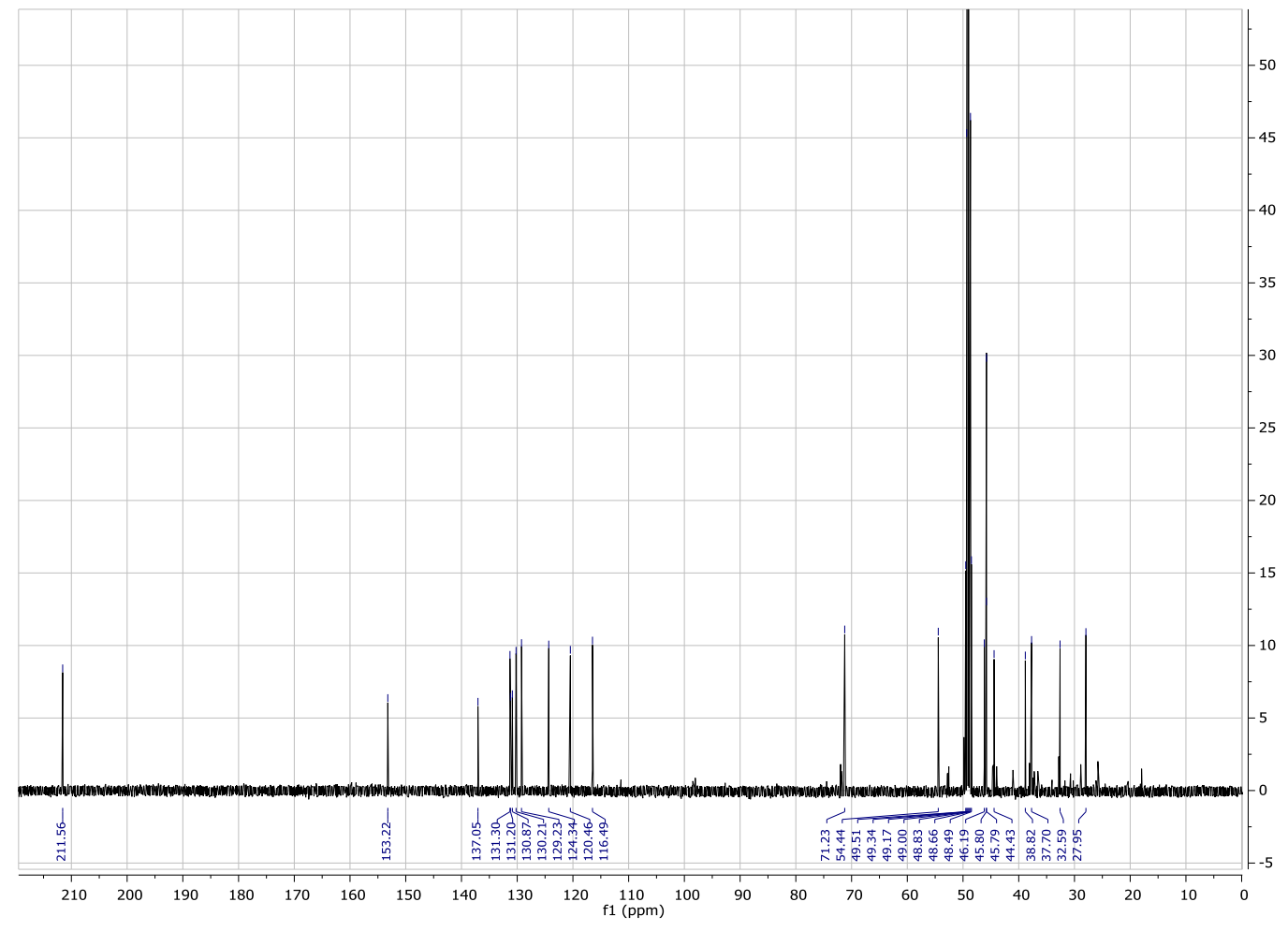


C)

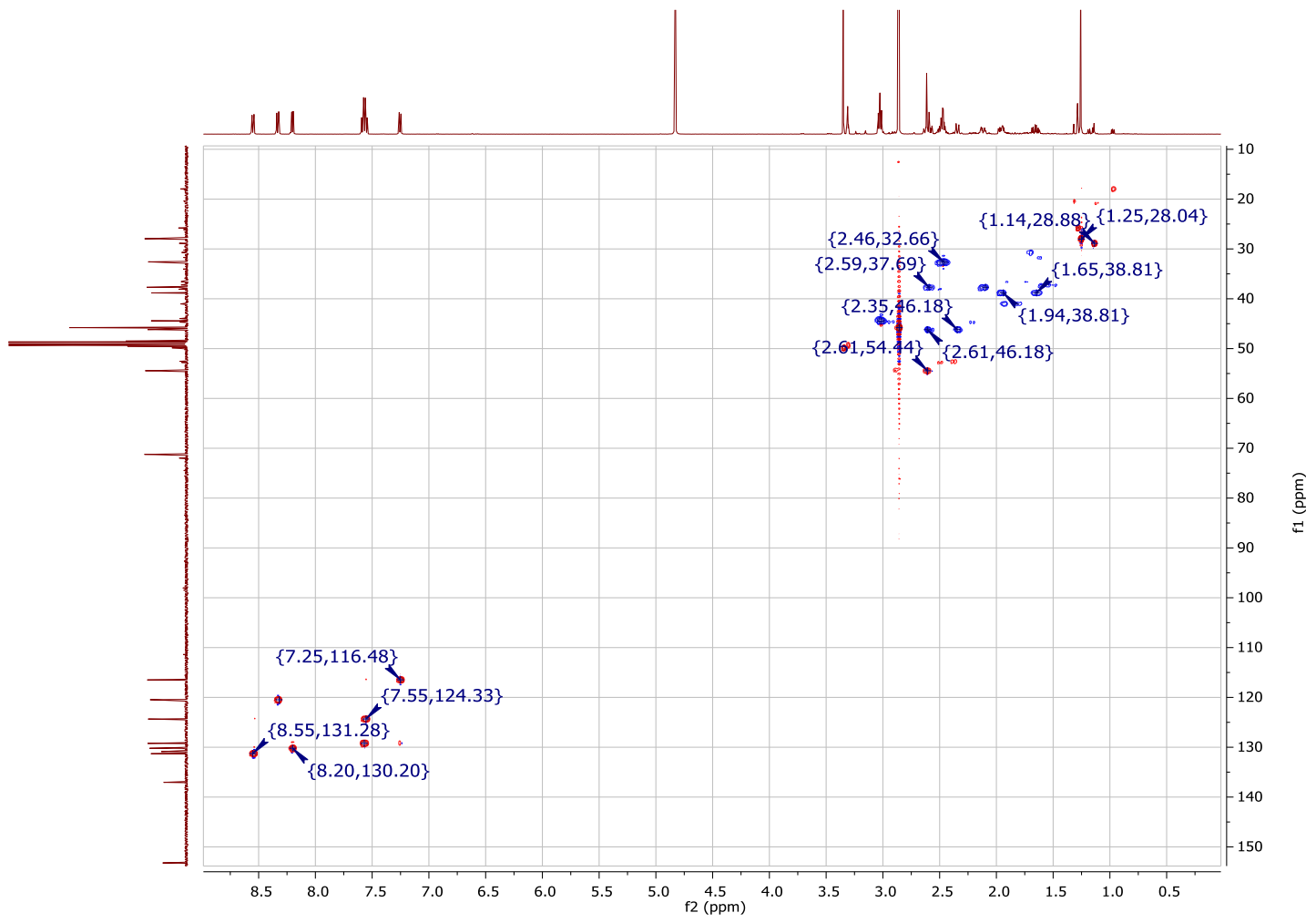

D)

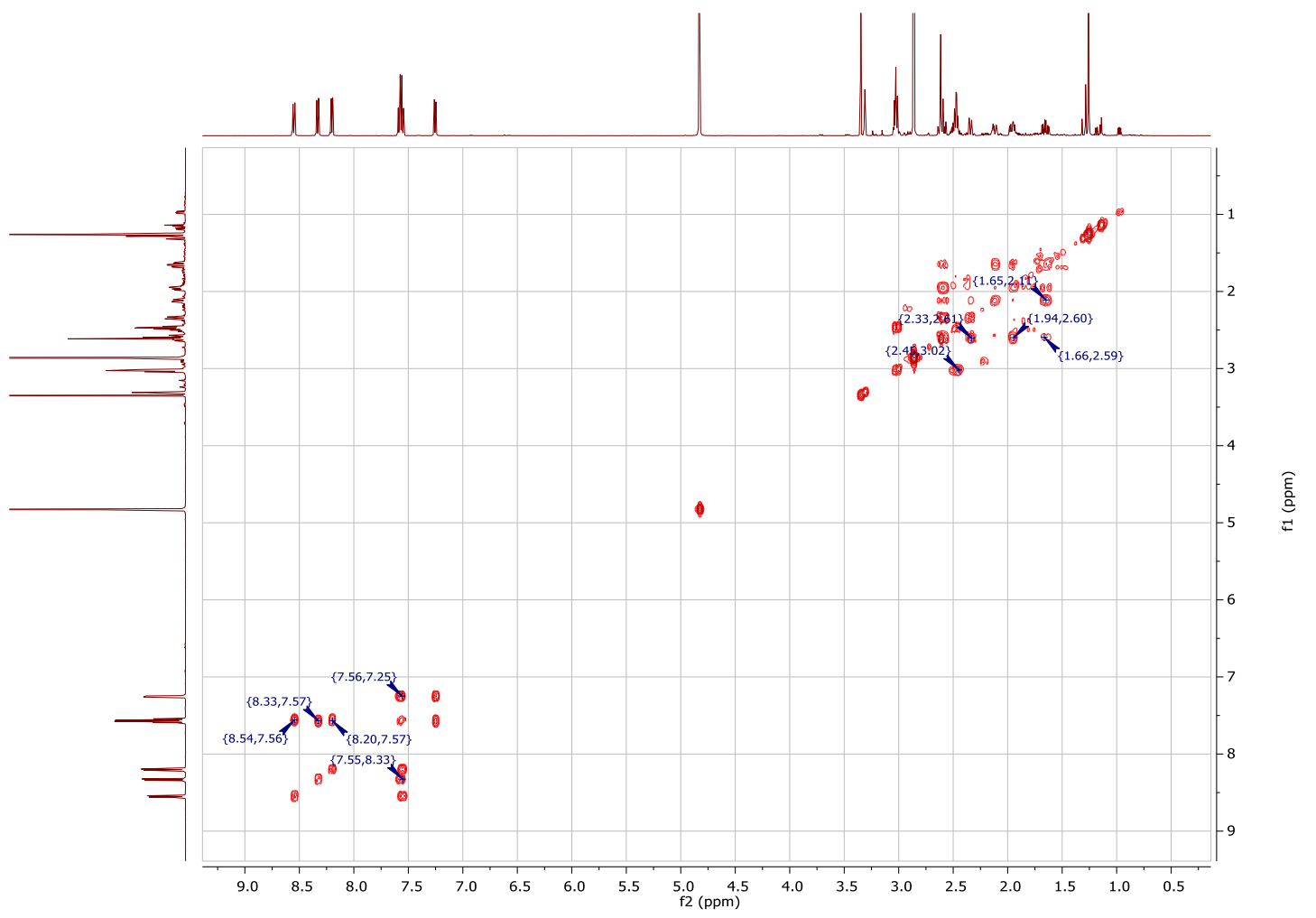


E)

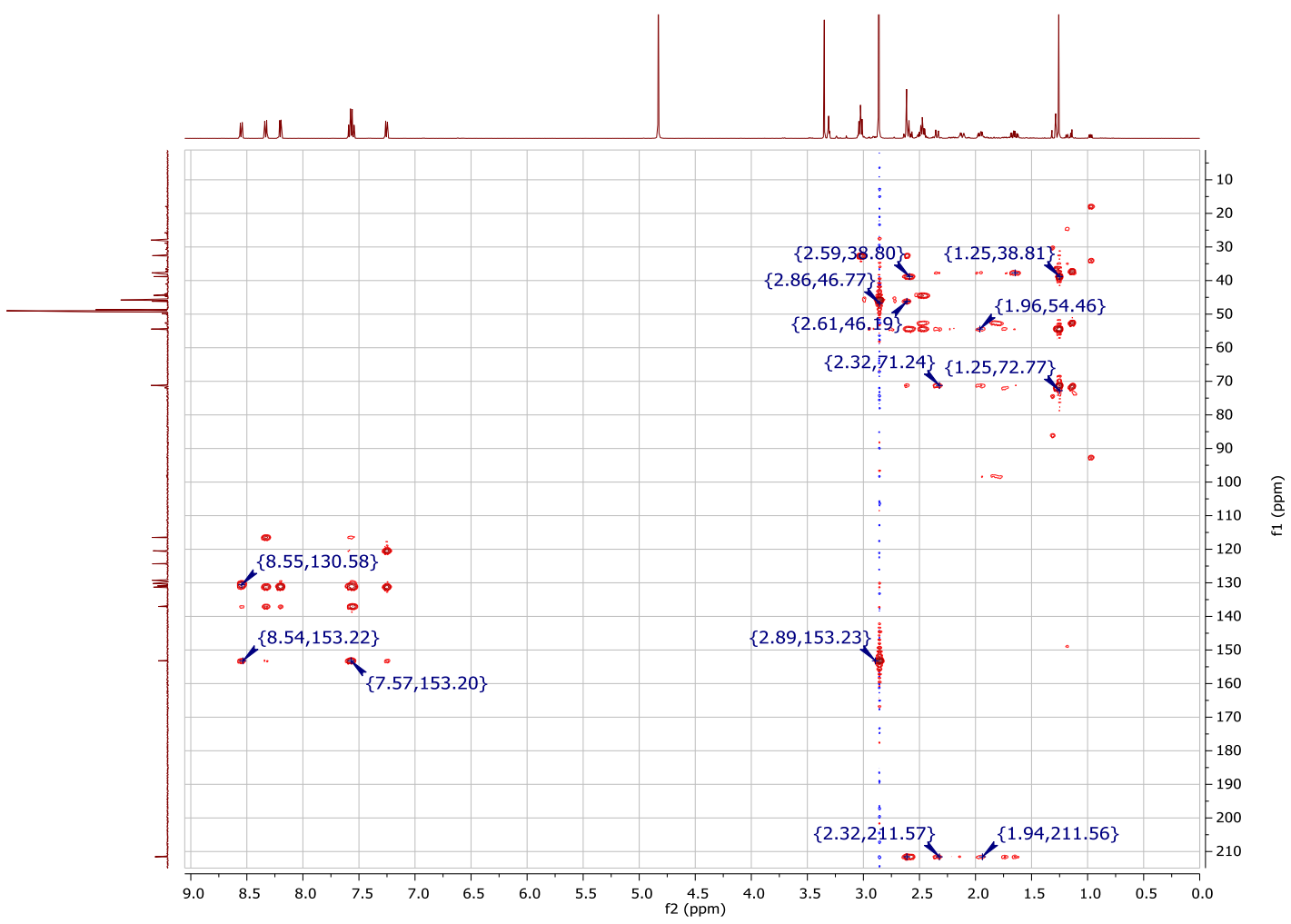

